TUMOUR IMMUNOLOGY

\section{Metastasis in action}

Using a surgically inserted 'window', researchers have been able to watch in real time metastatic melanoma cells as they spread to the lungs. This revealed that shear forces in the lung capillaries cause tumour cells to shed microparticles, which are taken up by a range of immune cells that influence the success of the metastases.

Their studies were prompted by the observation that immune cells in the lungs of mice with subcutaneous tumours became loaded with fragments of tumour cells before the appearance of lung micrometastases. So, to witness the arrival and fate of circulating tumour cells in the lungs, the authors performed intravital microscopy using a

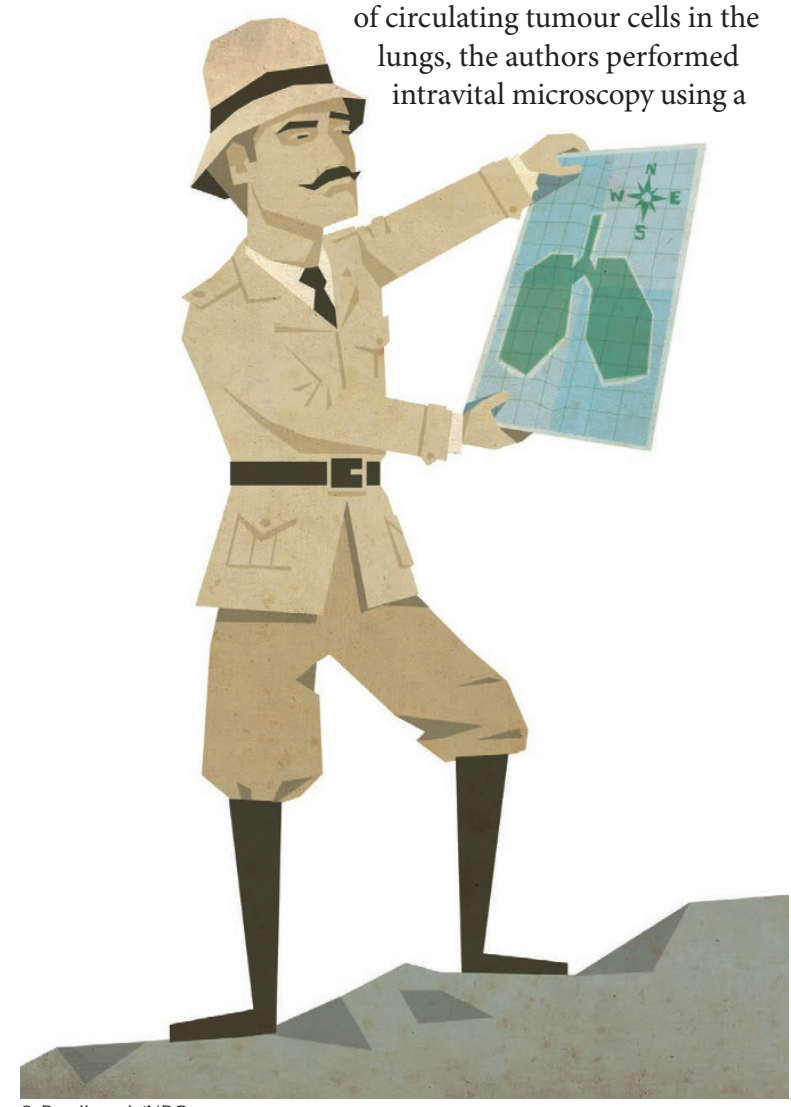

novel intercostal window. Within seconds of arriving in the lungs, fluorescently labelled $\left(\mathrm{ZsGreen}^{+}\right)$circulating tumour cells became lodged in capillaries and began to shed microparticles into the vasculature. These particles contained tumour cell cytoplasm (and were therefore termed cytoplasts), leaving behind the parental nucleated karyoplasts. Cytoplasts formed only in the presence of shear forces and could arrest and adhere to vascular walls or could migrate against vascular flow. Of interest, myeloid cells in the lungs were seen directly ingesting the cytoplasts but not karyoplasts: accordingly, ZsGreen ${ }^{+}$myeloid cells increased as free cytoplasts decreased.

A more detailed characterization of the tumour-ingesting myeloid cells showed that different myeloid cell types were involved in progressive waves. In the first wave (within 15 minutes of injection of ZsGreen ${ }^{+}$ tumour cells), neutrophils dominated in the uptake of cytoplasts. In the second wave, conventional monocytes became loaded with tumour-derived material, and the last wave (6-24 hours after injection) involved uptake by non-alveolar macrophages, patrolling monocytes and dendritic cells (DCs).

It has previously been shown that monocytes and monocytederived cells support successful early metastasis. This is consistent with findings in the current study showing that nascent metastases contained $\mathrm{ZsGreen}^{+}$non-motile myeloid cells and that some ZsGreen ${ }^{+}$ macrophages upregulated markers of activation. By contrast, the authors suggested that tumour-ingesting DCs might prime an antitumour $\mathrm{T}$ cell response. In support of this, injection of tumour cells expressing ZsGreen and an ovalbumin peptide resulted in the generation of clusters of $\mathrm{ZsGreen}^{+}$migratory CD103+ ${ }^{+} \mathrm{DCs}$ and ovalbumin-specific T cells in lung-draining lymph nodes.

CC-chemokine receptor 2 (CCR2) is thought to be important for the recruitment of myeloid cells to the lungs, so the authors studied $\mathrm{Ccr} 2^{-/-}$mice to better understand the functional roles of these cells in lung metastasis. Following tumour cell injection, $\mathrm{Ccr}^{-/-}$mice recruited fewer monocytes and macrophages to the lungs and developed fewer metastases. Instead, tumour-derived cytoplasts accumulated in lungresident $\mathrm{CD}_{103}{ }^{+} \mathrm{DCs}$, which supported a marked influx of $\mathrm{CD}^{+} \mathrm{T}$ cells into the lungs of $\mathrm{Ccr} 2^{-/-}$ mice compared with wild-type mice. This suggests that protective $\mathrm{CD}_{103}{ }^{+}$DCs compete with protumour macrophages to determine metastatic success; a hypothesis that was tested by temporarily depleting DCs in mice. Either following tumour cell injection or in the presence of a subdermal primary tumour, DC-depleted mice developed sixfold more lung metastases than non-depleted controls, which is consistent with a major role for DCs in restricting metastases.

So, watching metastasis in action has provided insight into the ways in which metastatic cells first interact with immune cells in the colonized tissue and how phagocyte populations compete to determine the outcome of metastatic seeding. Lucy Bird, Senior Editor, Nature Reviews Immunology

This article originally appeared in Nat. Rev. Immunol. (http://dx.doi.org/10.1038/nri.2016.45). 